\title{
El uso de cuadernillos de autoayuda en el Ménière atenúa el mareo
}

\author{
The Use of Booklet-Based Self-Management in Ménière attenuates dizziness
}

\section{Objetivo}

Evaluar la efectividad de manuales de autoayuda para la rehabilitación vestibular (RV) y de técnicas de control de síntomas (CS) para el manejo del mareo y el vértigo en la enfermedad de Méniere.

\section{Diseño}

Ensayo aleatorizado controlado.

\author{
Lugar \\ Escuela de Psicología de la Universidad de Southampton, \\ Inglaterra.
}

\section{Pacientes}

360 pacientes con diagnóstico de enfermedad de Ménière, con mareos o inestabilidad en los últimos 12 meses y sin crisis de vértigo severa durante las últimas seis semanas.

\section{Intervención}

Se aleatorizó a la población a tres grupos: 1) Manual de Educación en Rehabilitación Vestibular (GRV) con ejercicios de movimientos cefálicos repetitivos para promover una habituación neurológica y psicológica de los síntomas; 2) Manual de Control de Síntomas (GCS) con técnicas de relajación, respiración controlada y estrategias cognitivo-conductuales para reducir la amplificación de los síntomas debida a ansiedad; 3) grupo control (GC) al que se le informó que estaba incluido en una lista de espera.

\section{Medición de resultados principales}

El resultado primario fue el grado de mejoría subjetiva y capaci- dad a los seis meses. Los resultados secundarios fueron la adherencia al tratamiento, la severidad de los síntomas, la discapacidad, las creencias negativas sobre un ataque de vértigo y los síntomas de ansiedad y depresión.

\section{Resultados Principales}

El GRV y el GCS reportaron mayor mejoría que el GC a los seis meses (tabla 1). A los tres meses, el GRV mostró una reducción en los síntomas, ansiedad, discapacidad y creencias negativas sobre el mareo; el GCS redujo la discapacidad; y el GC no mostró ninguna mejoría.

Tabla 1: resultados de los manuales de autoayuda sobre el mareo a los seis meses.

\begin{tabular}{|c|c|c|c|}
\hline & $\begin{array}{l}\text { Gontrol } \\
n=120\end{array}$ & $\begin{array}{l}\text { Rehabilitación vestibular } \\
\qquad n=120\end{array}$ & $\begin{array}{c}\text { Control de síntomas } \\
n=120\end{array}$ \\
\hline Mejora subjetiva & $15,8 \%$ & \begin{tabular}{|l|l|}
$37,5 \%$ & RR: $2,37(1,48-3,80)$ \\
& NNT: $5(3-9)$
\end{tabular} & \begin{tabular}{|l|l|}
$39,2 \%$ & RR: $2,4 \quad(1,55-$ \\
& $3,95)$ NNT: 4 (3-8)
\end{tabular} \\
\hline $\begin{array}{l}\text { Promedio de puntaje } \\
\text { de capacidad (DE) }\end{array}$ & $1,17 \quad 2.34$ & $3,49 \pm 3,96$ & $3,26 \pm 3,49$ \\
\hline
\end{tabular}

\section{Conclusiones}

Los cuadernillos de autoayuda para el manejo de los síntomas en pacientes con enfermedad de Ménière son una herramienta efectiva, poco costosa y de sencilla implementación.

Palabras claves: enfermedad de Ménière, rehabilitación, educación al paciente. Key Words: Meniere's disease, rehabilitation, patient education.

\section{Comentario}

Como en todos los trastornos que deterioran la calidad de vida, la sola comprensión de los síntomas ayuda al paciente a manejarlos; y el saber qué es lo que debe esperar de una crisis disminuye la ansiedad y favorece el control del cuadro. Se piensa que el estrés es un factor disparador de crisis. Además es común en pacientes con vértigo recurrente (ej. enfermedad de Ménière) que sensaciones fisiológicas (como las que se tienen ante un giro brusco) le desencadenen el recuerdo de sus crisis y generen gran ansiedad y temor secundario. Por lo tanto, es fundamental enseñarles a controlar sus síntomas explicándoles lo que les sucederá en cada crisis y lo qué deberán realizar durante las mismas.

Sin embargo este tratamiento no actúa sobre el curso crónico de la enfermedad de Ménière. Durante cada crisis se destruyen receptores auditivos, lo que produce hipoacusia neurosensorial progresiva; y también se dañan receptores vestibulares, provocando inestabilidad residual crónica. Sobre estos trastornos específicos, la rehabilitación vestibular es una terapéutica de probada eficacia.

\section{Conclusiones del comentador}

En todo paciente con enfermedad de Ménière se debería realizar una combinación de estrategias, dándole las herramientas para un adecuado control de la ansiedad que genera esta enfermedad y rehabilitando su sistema vestibular para conseguir un mayor grado de estabilidad.

Ver glosario*

Guillermo Videla [, Sección de Trastornos de Marcha y Equilibrio, Servicio de Neurología. Hospital Italiano de Buenos Aires. ]

Videla G. El uso de cuadernillos de autoayuda en el Ménière atenúa el mareo. Evid. actual. práct. ambul; 10(3): 74, May-Jun 2007. Yardley L. Evaluation of Booklet-Based Self-Management of Symptoms in Ménière Disease: A Randomized Controlled Trial. Psychosom Med. 2006; 68(5):762-9PMID: 17012531

Referencia

1. Comité on Hearing and Equilibrium. Guidelines for the diagnosis and evaluation of therapy in Meniere's disease. Otolaryngol Head Neck Surg 1995; 113:181-5.

2. Gant ND. Psychosocial challenges faced by persons with Meniere's disease. J Appl Rehabil Couns 1997:28:40-9.

3. Yardley L, et.al. Factors associated with quality of life in Meniere's disease. Clin Otolaryngol 2003;28:436-41.

4. Clendaniel, et. al. Vestibular rehabilitation strategies in Meniere's disease. Otolaryngol Clin North Am 1997;30:1145-58.

5. Gottshall K, et. al. The role of vestibular rehabilitation in the treatment of Meniere's disease. Otolaryngol Head Neck Surg 2005;133:326-8. 